guided fine needle biopsy (FNB) was performed. An ERCP was performed in the same session with insertion of a double pigtail stent into the CBD. However over the following days the patient remained icteric.

Histological samples were reviewed by both the adult and paediatric histopathology departments. The FNB specimens showed benign acinar groups and predominantly neutrophilic inflammatory infiltrate, acinar inflammation and fibrosis. There was no positive IgG4 staining in the few plasma cells.

Once prednisolone was commenced the patient clinically and biochemically improved. A diagnosis of acute autoimmune pancreatitis was made.

Discussion P-AIP presents with very different clinical and biochemical signs compared to adult type. This case report discusses the challenging issue of diagnosing P-AIP in Ireland and worldwide. Currently in Ireland there is no dedicated paediatric EUS service. International expert group consensus statements recommend that ideally P-AIP be confirmed by welldescribed and pathognomonic histopathological features in a pancreatic biopsy. This case report demonstrates how paediatric patients can benefit from adult subspecialist $\mathrm{PB}$ expertise allowing further radiological and histopathological information to achieve a definitive diagnosis of P-AIP. It is important that paediatricians and paediatric surgeons in Ireland and abroad be aware of the benefits of this collaboration in the P-AIP setting.

\section{GP184 THE GERDQ QUESTIONNAIRE FOR DIAGNOSIS OF GASTRO-ESOPHAGEAL REFLUX DISEASE IN CHILDREN}

Vladimir Shcherbak*, Tatiana Aksenova, Natalia Shcherbak. Chita State Medical Academy, Chita, Russian Federation

\subsection{6/archdischild-2019-epa.245}

Background In recent years, GerdQ (Gastro-esophageal reflux disease Questionnaire) had been used in adults (R. Jones, J. Den, N. Vakil et al), but it has not been used in children before.

The objective is to determine the validity of the GerdQ questionnaire for the diagnosis of gastro-esophageal reflux disease (GERD) in children with symptoms suggestive of GERD. Methods 63 patients aged 12-17 years with gastroenterological complaints were examined. At first the patients answered GerdQ questions, and then they underwent $\mathrm{pH}$-metry and esophagofibrogastroscopy. According to the developers of the questionnaire, with a score of 8 or more, the likelihood of GERD is high, and 7 and lower is unlikely

Results The following data were obtained from the questionnaire: $0-2$ points - 4 patients (6.4\%), 3-7 points - 46 (73.0\%), 8-10 points - 11 (17.4\%), 11-18 points - 2 patients (3.2\%).

After instrumental examination among 13 patients with scores $\geq 8$ GERD was diagnosed in $9(69.2 \%)$, among 50 children with scores $<8$ GERD was excluded in 48 (96\%). The sensitivity of the GerdQ questionnaire in children was $69.2 \%$ (95\% CI: 53-76), specificity - 96\% (95\% CI: 71-97), which allows recommending it in a wide practice.

Patients with mild complaints may be prescribed a trial treatment with proton pump inhibitors already at the stage of primary treatment, without the use of additional instrumental diagnostic methods. However, this approach is fraught with certain difficulties associated with a subjective assessment of complaints, both by the patient and by the doctor. For example, patients with severe erosive-ulcerative lesions, metaplasia and dysplasia of the esophageal mucosa may have almost no clinical symptoms, whereas in other cases the abundance of the most diverse complaints actively presented by the patient is not accompanied by endoscopic changes in the mucous lining. In children, the frequency of metaplasia and dysplasia is not so great, although its significance should not be minimized. A reasonable combination of questionnaire and instrumental examination seems reasonable.

Conclusions GerdQ is a useful complementary method for the diagnosis of GERD in children. It could reduce the need for pH-metry and esophagofibrogastroscopy.

\section{GP185 THE DIAGNOSTIC AND PROGNOSTIC VALUE OF THE SHORT-CHAIN FATTY ACIDS IN ROTAVIRUS DIARRHEAS IN INFANTS}

Bayaz Babayeva*. Azerbaijan Medical University, Baku, Azerbaijan

\subsection{6/archdischild-2019-epa.246}

Introduction Acute intestinal infections have occupied a leading place in the infectious pathology in children so far. The condition of intestinal microbiocenosis is one of the factors that determine the severity of intestinal infection, its duration, the outcome and the timing of rehabilitation from the pathogen. One of the very promising techniques for evaluating intestinal microflora in health and disease is the determination of the metabolic activity of the intestinal microflora from the spectrum of short-chain fatty acids (SCFAs). In various diseases of the gastrointestinal tract, including viral diarrhea, the process of formation, absorption and utilization of SCFAs is disrupted, so their concentrations in feces change. The aim of our research was to specify the content and profile of metabolites of the normal intestinal microflora, as well as to scrutinize their diagnostic and prognostic significance for assessing the severity of rotavirus infection in infants.

Methods We examined 64 infants with rotavirus infection who were hospitalized in the Pediatrics Research Institute. The presence of rotavirus in feces was confirmed by the method of immune enzyme analysis ELISA. 30 healthy children were in the control group. The evaluation of the levels of (SCFAs) and their overall level was carried out by using the method of gas-liquid chromatography.

Results In the acute period of rotavirus infection, there was a significant decrease in the content of acetic $[2.478 \pm 0.05$ $\mathrm{mg} / \mathrm{ml}, \mathrm{p}<0.001]$, propionic $[0.545 \pm 0.019 \mathrm{mg} / \mathrm{ml} \mathrm{p}$ $<0.001]$, butyric $[0.489 \pm 0.019 \mathrm{mg} / \mathrm{ml} \mathrm{p}<0.001]$ and valerian acids $[0.075 \pm 0.001 \mathrm{mg} / \mathrm{ml} \mathrm{p}<0.001]$, as well as the general level of metabolites $[3.807 \pm 0.076 \mathrm{mg} / \mathrm{ml} \mathrm{p}<0.001]$. An inverse correlation was found between the degree of the severity of the disease and concentrations of acetic $(p<0.05)$, propionic ( $\mathrm{p}<0.05$ ), butyric acids $(\mathrm{p}<0.01)$, as well as the overall level of metabolites $(\mathrm{p}<0.05)$.

Conclusion Our studies have shown that the determination of the metabolic activity of intestinal microflora from the levels and spectra of SCFAs is important for the assessment of microecological bowel disorders in infants with viral diarrhea which can serve as an additional prognostic and diagnostic criterion for the severity of the course of the disease. 